\title{
Estimating excess mortality in people with cancer and multimorbidity in the COVID-19 emergency
}

\author{
Alvina G. Lai, Ph.D. ${ }^{1,2, \mathbb{}}$, Laura Pasea, Ph.D. ${ }^{1,2^{*}}$, Amitava Banerjee, DPhil ${ }^{1,2,3^{*}}$, Spiros Denaxas, Ph.D. ${ }^{1,2,6,7}$, Michail Katsoulis, \\ Ph.D. ${ }^{1,2}$, Wai Hoong Chang, MSc ${ }^{1,2}$, Bryan Williams, Ph.D. ${ }^{4,5}, 6$, Deenan Pillay, Ph.D. ${ }^{8}$, Mahdad Noursadeghi, Ph.D. ${ }^{8}$, David \\ Linch, FMedSci ${ }^{6,9}$, Derralynn Hughes, FRCPath ${ }^{10,11}$, Martin D. Forster, Ph.D. ${ }^{4,10}$, Clare Turnbull, Ph.D. ${ }^{12}$, Natalie K. Fitzpatrick, \\ MSc $^{1,2}$, Kathryn Boyd, MD ${ }^{13}$, Graham R. Foster, Ph.D. ${ }^{14}$, DATA-CAN ${ }^{15}$, Matt Cooper, Ph.D. ${ }^{15}$, Monica Jones, PGDip ${ }^{15}$, Kathy \\ Pritchard-Jones, FMedSci ${ }^{15,16,17,18}$, Richard Sullivan, Ph.D. ${ }^{19}$, Geoff Hall, Ph.D. ${ }^{15,20,21}$, Charlie Davie, FRCP ${ }^{11,15,16}$, Mark \\ Lawler, Ph.D. ${ }^{152}$, and Harry Hemingway, FMedSci ${ }^{1,2,6, \infty}$

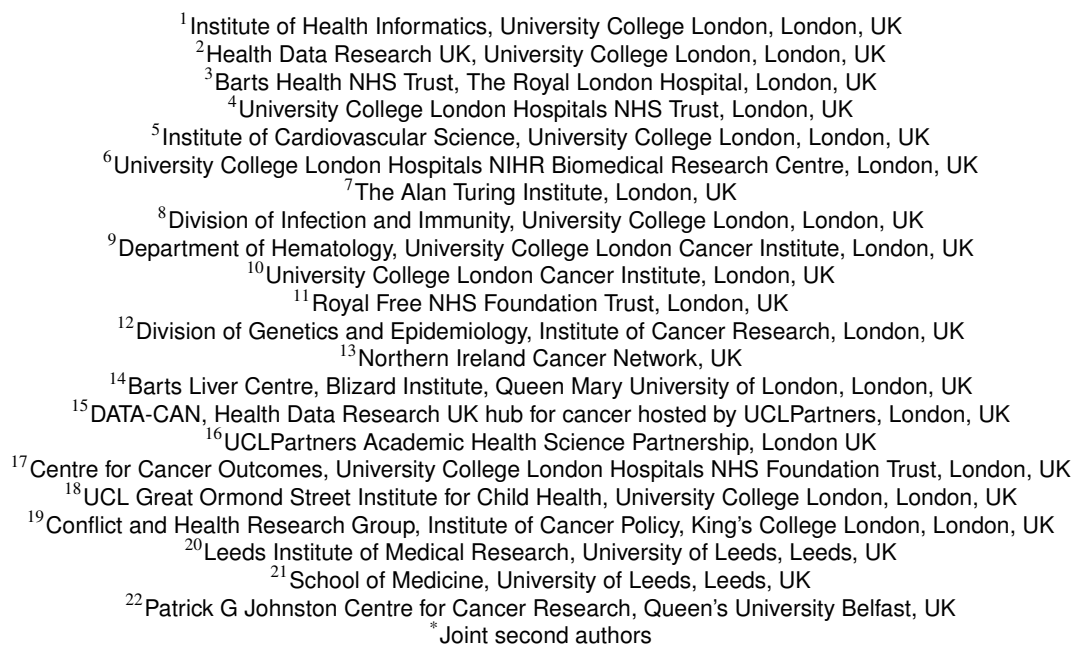

Background: Cancer and multiple non-cancer conditions are considered by the Centers for Disease Control and Prevention (CDC) as high risk conditions in the COVID-19 emergency. Professional societies have recommended changes in cancer service provision to minimize COVID-19 risks to cancer patients and health care workers. However, we do not know the extent to which cancer patients, in whom multi-morbidity is common, may be at higher overall risk of mortality as a net result of multiple factors including COVID-19 infection, changes in health services, and socioeconomic factors.

Methods: We report multi-center, weekly cancer diagnostic referrals and chemotherapy treatments until April 2020 in England and Northern Ireland. We analyzed populationbased health records from 3,862,012 adults in England to estimate 1-year mortality in 24 cancer sites and 15 noncancer comorbidity clusters (40 conditions) recognized by CDC as high-risk. We estimated overall (direct and indirect) effects of COVID-19 emergency on mortality under different Relative Impact of the Emergency (RIE) and different Proportions of the population Affected by the Emergency (PAE). We applied the same model to the US, using Surveillance, Epidemiology, and End Results (SEER) program data.

Results: Weekly data until April 2020 demonstrate significant falls in admissions for chemotherapy (45-66\% reduction) and urgent referrals for early cancer diagnosis $\mathbf{( 7 0 - 8 9 \%}$ reduction), compared to pre-emergency levels. Under conservative assumptions of the emergency affecting only people with newly diagnosed cancer (incident cases) at COVID19 PAE of $40 \%$, and an RIE of 1.5 , the model estimated 6,270 excess deaths at 1 year in England and 33,890 excess deaths in the US. In England, the proportion of patients with incident cancer with $\geq 1$ comorbidity was $65.2 \%$. The number of comorbidities was strongly associated with cancer mortality risk. Across a range of model assumptions, and across incident and prevalent cancer cases, $78 \%$ of excess deaths occur in cancer patients with $\geq 1$ comorbidity.

Conclusion: We provide the first estimates of potential excess mortality among people with cancer and multimorbidity due to the COVID-19 emergency and demonstrate dramatic changes in cancer services. To better inform prioritization of cancer care and guide policy change, there is an urgent need for weekly data on cause-specific excess mortality, cancer diagnosis and treatment provision and better intelligence on the use of effective treatments for comorbidities.

Correspondence: alvina.lai@ucl.ac.uk / h.hemingway@ucl.ac.uk

\section{Introduction}

The excess risk of death in people living with cancer during the COVID-19 emergency may be due not only NOTE: This preprint reports new research that has not been certified by peer review and should not be used to guide clinical practice. 
to COVID-19 infection, but also to the unintended health consequences of changes in health service provision, the physical or psychological effects of social distancing, and economic upheaval. Recent national evidence suggests that there is an excess of deaths, both in those infected with SARS-CoV-2, and in those with no infection(1). Optimal cancer care must balance the need to protect patients from COVID-19 infection, with continued access to early diagnosis and potentially curative treatment $(2,3)$. Professional associations in the US(4-6), UK(7-9) and Europe(9) have recommended revising cancer care activities for triaging of systemic anti-cancer treatment, surgery and risk-adapted radiotherapy(10). All elective surgery has been postponed in the $\mathrm{UK}(8,9)$. In the US, more than a quarter of patients with cancer reported a delay in their cancer treatment in April 2020 because of COVID-19(4). Despite these recommendations for changes in healthcare services, there is a lack of near real-time data quantifying the extent of disruption due to reconfiguration in service delivery for cancer patients.

Existing publications on cancer and COVID-19 have been limited to small case series $(11,12)$. The US Centers for Disease Control and Prevention (CDC) and Public Health England (PHE) have identified patients with specific malignant and non-malignant conditions as at greater risk of developing severe illness from COVID-19 exposure(13-15). Patients with cancer commonly have other conditions considered to further increase their COVID-19 risk; multimorbidity in cancer is an increasing clinical concern(16, 17). Oncologists, internists and family physicians need to balance the requirement to encourage people to socially isolate, with their need to access hospital services to ensure the most effective cancer care. There is a lack of evidence on pan-cancer estimation of mortality risks according to type and number of multimorbid conditions.

Our objectives were to: 1) quantify changes in cancer care activities in near real-time from weekly multi-center hospital data; 2) estimate the prevalence, incidence and background (pre-COVID-19) 1-year mortality risk across 24 site-specific cancers; 3) estimate the prevalence of 15 CDC- and PHErelevant co-occurring condition clusters (multimorbidity) by cancer site and their association with excess mortality and 4) estimate overall excess deaths due to the COVID-19 pandemic over a 1-year period, based on different Proportion of the population Affected by the Emergency (PAE) and Relative Impact of the Emergency (RIE), using a previously published model(18).

\section{Methods}

Weekly information on cancer care. Through DATACAN, the UK National Health Data Research Hub for Cancer(19), we obtained weekly returns for urgent cancer referrals for early diagnosis and chemotherapy attendances (from 2018 to most current data) from hospitals in Leeds, London and Northern Ireland.
Patient cohort. We used population-based electronic health records in England from primary care data linked to the Office for National Statistics(ONS) death registration, using the open-access CALIBER resource $(20,21)$. The study population consisted of $3,862,012$ adults aged $\geq 30$ years, registered with a general practice from 1 January 1997 to 1 January 2017 with at least one year of follow-up data.

Electronic health record phenotype definitions of diseases and risk factors relevant to COVID-19 are available at https://caliberresearch.org/portal and have previously been validated(22-25). Further details are in supplementary methods. In brief, phenotypes are based on hospital and primary care information recorded in primary care, using the Read clinical terminology(version 2). We defined non-fatal, incident and prevalent cases of cancer across 24 primary cancer sites according to previously validated CALIBER electronic health record phenotypes, which included: biliary tract, bladder, bone, brain, breast, cervix, colon-rectum, Hodgkin's lymphoma, kidney, leukemia, liver, lung, melanoma, multiple myeloma, non-Hodgkin's lymphoma, esophagus, oropharynx, ovary, pancreas, prostate, stomach, testis, thyroid and uterus(26). We defined cancers as prevalent (diagnosed at any time prior to baseline) and incident if they occurred during follow-up. We defined 15 comorbid conditions or condition clusters involving 40 individual conditions defined by the Centers for Disease Control (CDC) or Public Health England (PHE) as associated with poor outcomes caused by severe COVID-19 infection.

Analyses. For full details of the analyses, see supplementary methods. Briefly, we estimated incidence rates per 100,000 person years and 1-year mortality in our study population. Estimates were used to calculate the excess deaths in cancer alone and cancer plus comorbidities, based on three levels of PAE $(10 \%, 40 \%$ and $80 \%)$ and four RIE scenarios associated with COVID-19(1.2, 1.5, 2.0 and 3.0) (additional details on plausible choice of PAE and RIE are presented in the discussion). We presented all results in Figures 2, 5, S4 and S8, but in order to represent a plausible, likely conservative, scenario for the current emergency, we chose to highlight data for a PAE of $40 \%$ and a RIE of 1.5 , with a range of 1.2-2 in the results text section of the manuscript. We applied our model to US population using publicly available data from the US from the Surveillance, Epidemiology, and End Results (SEER) program on incidence and 1 year mortality (taken as 1 minus the reported survival(27)). We considered mortality data from SEER for individuals aged 40-64, 65-74 and 75+.

\section{Results}

Real-time weekly hospital data for urgent cancer referrals and chemotherapy attendances in England and Northern Ireland. We observed that a majority of patients with cancer or suspected cancer are not accessing healthcare services. Major declines in chemotherapy attendances $(45 \%$, $66 \%, 66 \%$ and $63 \%$ reduction; average $=60 \%$ ) and urgent 
medRxiv preprint doi: https://doi.org/10.1101/2020.05.27.20083287; this version posted June 1, 2020. The copyright holder for this preprint (which was not certified by peer review) is the author/funder, who has granted medRxiv a license to display the preprint in perpetuity. It is made available under a CC-BY-NC-ND 4.0 International license .
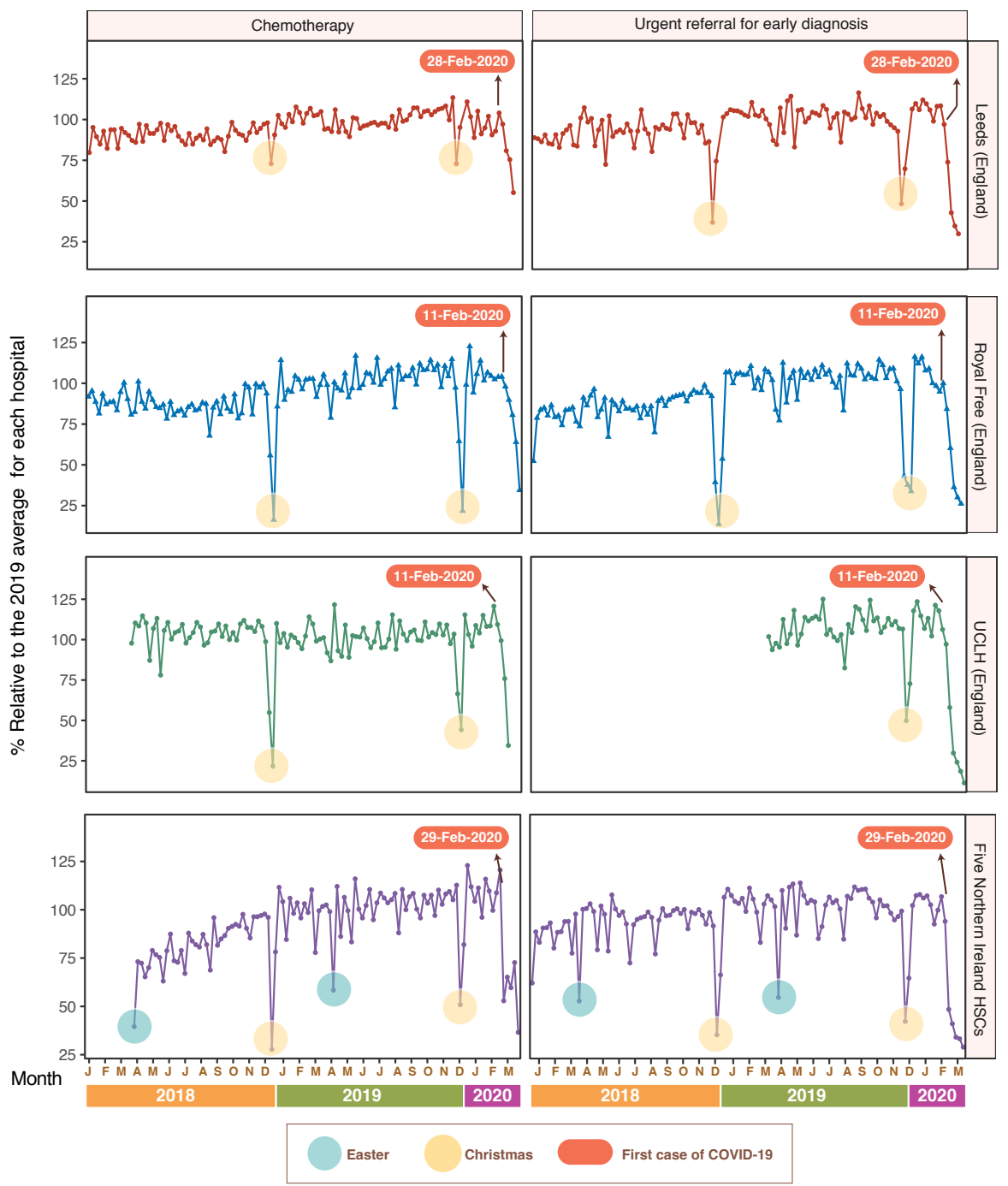

Fig. 1. Weekly hospital data on changes in urgent referrals and chemotherapy clinic attendance from eight hospitals in the UK. The data for Northern Ireland includes five Health and Social Care Trusts (HSCs) that cover all health service provision in Northern Ireland: Belfast HSC, Northern HSC, South Eastern HSC, Southern HSC and Western HSC.

cancer referrals for early diagnosis $(70 \%, 74 \%, 89 \%$ and $71 \%$ reduction; average $=76 \%$ ) were observed in eight hospitals across the UK: England (Leeds Teaching Hospitals NHS Trust, Royal Free Hospital and University College London Hospitals) and Northern Ireland (all five Health and Social Care Trusts) respectively, compared to pre-emergency levels (Figure 1). These data signal the need to identify patientspecific risk factors (cancer and multimorbidity) to facilitate prioritization of service provision during the pandemic.

Prevalence, incidence and background (pre-COVID-19) 1-year mortality for $\mathbf{2 4}$ cancers. The overall prevalence of any of the 24 cancers was $3.1 \%(117,978 / 3,862,012)$ in CALIBER (England), (Table S1). Prevalence of the 24 cancers by age, sex and year is shown in Figure S1. The age-adjusted incidence rates of the 24 cancers estimated in CALIBER was 635 per 100000 (compared with estimates from International Agency for Research on Cancer (IARC, UK) of 590 per 100 000) (Figure S2A). The 1-year mortality across
24 cancer sites was, as anticipated, higher among incident cases of cancer, compared to prevalent cases (Figure S3).

Estimates of excess COVID-19-related deaths by cancer site in England. When considering incident cancers in England, at COVID-19 PAE of 40\%, we estimated 2,509, 6270 and 12,543 excess deaths at RIE of 1.2, 1.5, and 2 respectively (Figure $2 \mathrm{~A}$ ). Higher numbers were seen among prevalent cancers than incident cancers when estimating absolute excess deaths, since the number of prevalent cases were higher than the number of incident cases observed over 1 year, e.g., for PAE 40\%, we observed 11,645 excess deaths (range 4,655-23,287) at RIE of 1.5 (range 1.2-2) (Figure S4). When considering both prevalent and incident cancers together at COVID-19 PAE of 40\%, we estimated 17,915 excess deaths (range 7,164-35,830) at RIE of 1.5 (range 1.2-2).

Estimates of excess COVID-19-related deaths by cancer site for incident cases in US. We found broadly comparable 
medRxiv preprint doi: https://doi.org/10.1101/2020.05.27.20083287; this version posted June 1, 2020. The copyright holder for this preprint (which was not certified by peer review) is the author/funder, who has granted medRxiv a license to display the preprint in perpetuity. It is made available under a CC-BY-NC-ND 4.0 International license .

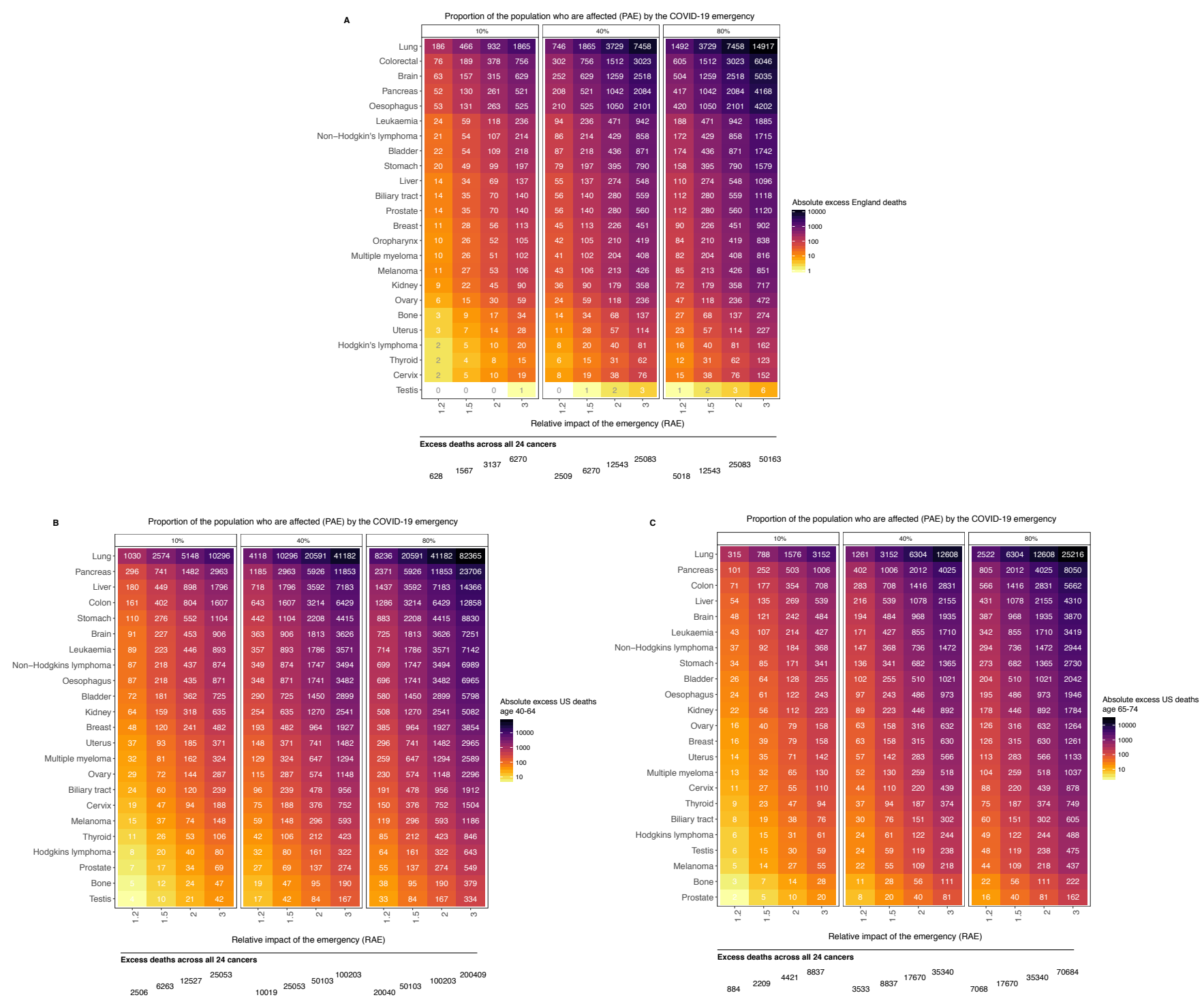

Fig. 2. Estimated number of excess deaths at 1 year due to the COVID-19 emergency by cancer site for incident cases (A) scaled up to the population of England aged $30+$ consisting of 35 million individuals using England (CALIBER) mortality estimates, (B) scaled up to the population of US aged 40-64 consisting of 208 million individuals using US (SEER) mortality estimates for this age range and (C) scaled up to the population of US aged 65-74 consisting of 61 million individuals using US (SEER) mortality estimates for this age range.

1-year mortality among incident cancers across cancer sites in England and the US (Figure S2B). For the US, based on SEER incidence and 1-year mortality for individuals aged 40-64, at COVID-19 PAE of $40 \%$, we estimated 10,019, 25,053 and 50,103 excess deaths at RIE of 1.2, 1.5 and 2 respectively, when extrapolating to the US population of this age group(208,284,801 individuals) (Figure 2B). Using mortality estimates for individuals aged $65-74$, at COVID-19 PAE of 40\%, we estimated 3,533, 8,837 and 17,679 excess deaths at RIE of $1.2,1.5$ and 2 respectively, when extrapolating to the US population of this age group $(61,346,445$ individuals) (Figure 2C). For a PAE of $40 \%$ and RIE of 1.5 (range 1.2-2), we estimated 33,890 excess deaths (range 13,552-67,782) in individuals older than age 40.
19 risk. Across all cancers, the proportions of $0,1,2$ and $3+$ comorbidities were $51.8 \%, 25.2 \%, 14.2 \%$ and $8.8 \%$ respectively for prevalent cancers. Comorbidities were common in people with cancer; e.g., hypertension (34,696 [19.0\%]), CVD (23,532 [12.9\%]), CKD (9,530 [5.2\%]) and obesity (9,491 [5.2\%]) (Figure 3). Prevalence of comorbidities in some cancers differed from the condition's prevalence in the overall population. For example, COPD (3.0\%) compared to CVD (12.9\%) is a relatively uncommon comorbidity in patients with cancer, except for lung cancer where patients had a higher than background COPD prevalence (25.7\%) (Figure 3). Conversely, a 'metabolic phenotype' was particularly common in uterine cancer, where a relatively large proportion of patients had hypertension (48.8\%), CVD (26.2\%), obesity (14.1\%) and type-2 diabetes (9.3\%) (Figure 3).

\section{Proportion of 15 comorbidity clusters relevant to COVID-}


medRxiv preprint doi: https://doi.org/10.1101/2020.05.27.20083287; this version posted June 1, 2020. The copyright holder for this preprint (which was not certified by peer review) is the author/funder, who has granted medRxiv a license to display the preprint in perpetuity. It is made available under a CC-BY-NC-ND 4.0 International license .

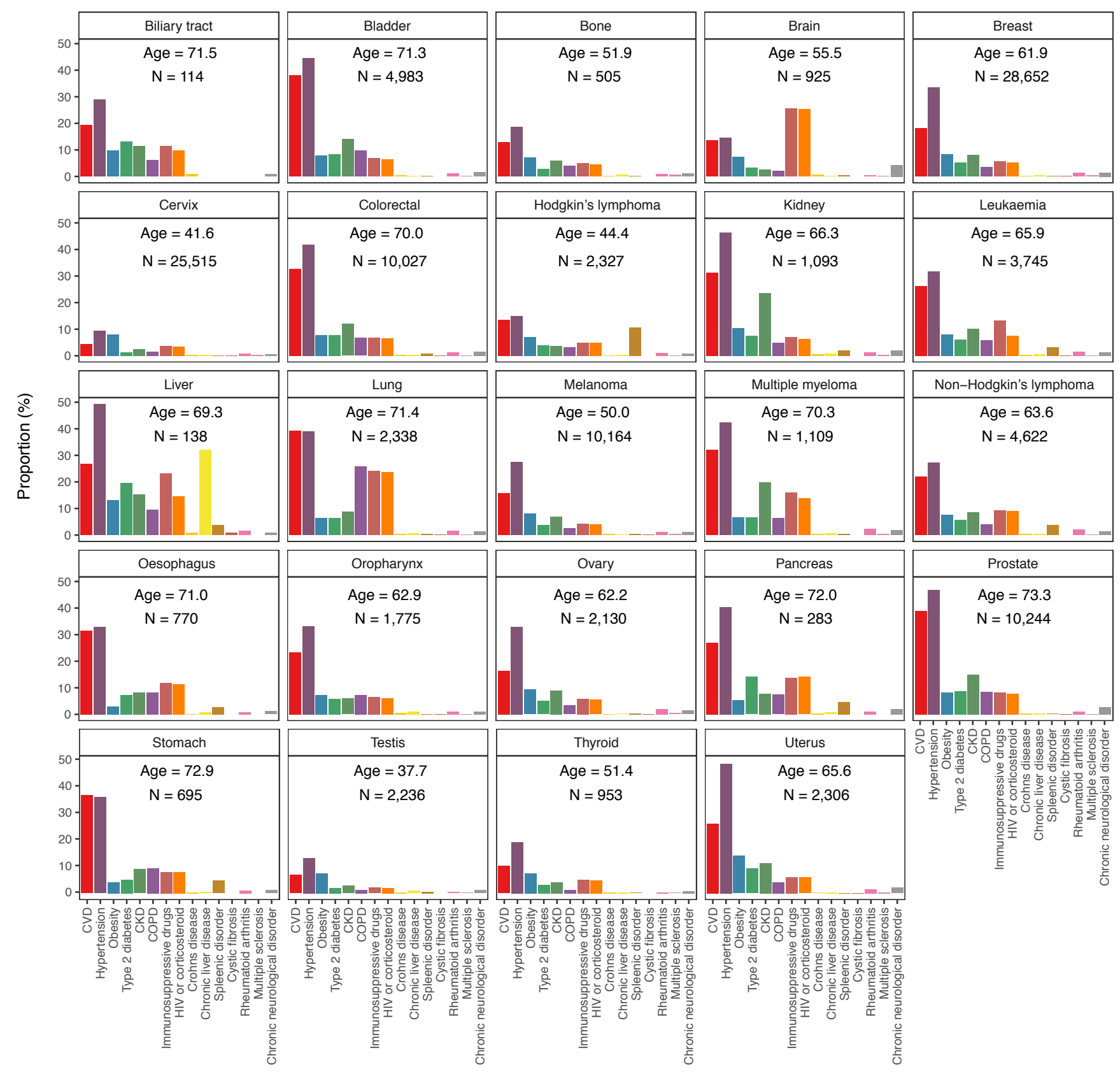

Fig. 3. Proportion of patients with any of the 15 comorbidity clusters by cancer site for prevalent cases ( $N=117,978$ ) in a population of $3,862,012$ adults in England (CALIBER). Age indicates mean age at diagnosis.

Number of comorbid conditions and 1-year mortality in incident cancers. The proportions of individuals with 0 , 1,2 and $3+$ comorbidities for incident cancers were $34.8 \%$, $25.6 \%, 20.5 \%$ and $19.2 \%$ respectively (Figure $4 \mathrm{~A}$ ). We found in incident cases that multimorbidity ( $\geq 3$ vs 0 conditions) was associated with a further increase in 1-year mortality. Cancers of the pancreas $(65.7 \%$ vs. $80.1 \%)$, biliary tract (58.6\% vs. $64.8 \%)$, lung $(51.9 \%$ vs. $60.9 \%)$, brain (46.4\% vs. $80.9 \%)$ and stomach (42.4\% vs. $48.3 \%)$ exhibited the most pronounced effects (Figure 4B, Figure S6). Consistent findings were found for prevalent cancer cases (Figure S5, Figure S7)
Excess 1-year COVID-19-related deaths by cancer site and number of comorbid conditions in England. For incident cancers, when considering COVID-19 PAE of $40 \%$ and RIE of 1.5, we observed 1,210, 1,509, 1,572 and 1,983 excess deaths in individuals with $0,1,2$ and $3+$ non-cancer comorbidities; 5,064 (80.7\%) of these deaths occur in patients with $\geq 1$ comorbidities (Figure 5). When considering COVID-19 PAE of $40 \%$ and RIE of 1.5 for prevalent cancers, we observed 2,724, 3,480, 2,955 and 2,558 excess deaths in individuals with $0,1,2$ and $3+$ non-cancer comorbidities; 8,993 (76.8\%) of these deaths occur in patients with $\geq 1$ comorbidities (Figure S8). When considering both prevalent and 
A

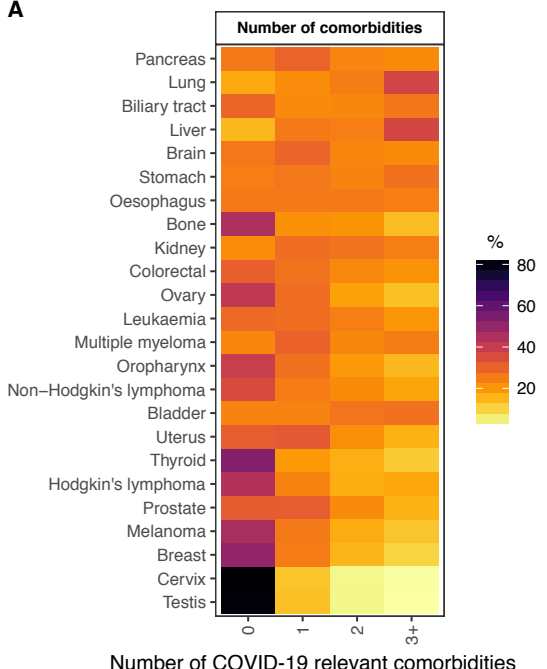

B

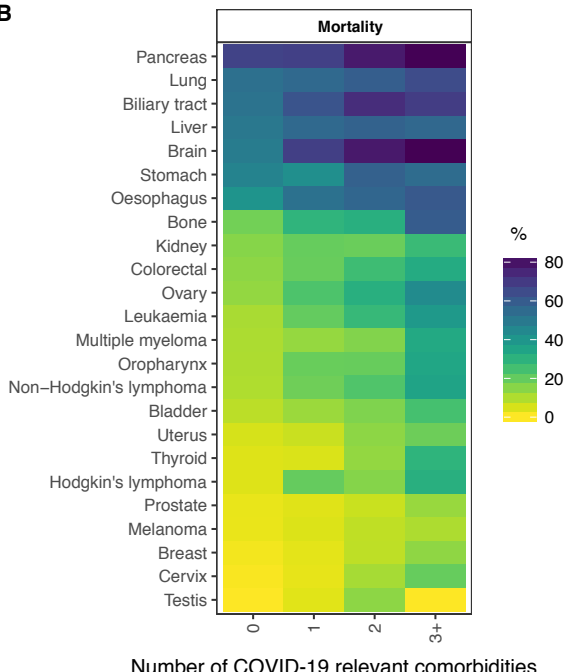

Fig. 4. Comorbidity clusters relevant for COVID-19 risk in incident cases in England (CALIBER). (A) Proportion of individuals with 0, 1, 2 and 3+ comorbidities by cancer site. (B) 1-year mortality in individuals with 0, 1,2 and 3+comorbidities by cancer site.

incident cancers together at COVID-19 PAE of 40\%, we estimated 17,991 excess deaths at RIE of $1.5 ; 78.1 \%$ of these deaths occur in patients with $\geq 1$ comorbidities.

\section{Discussion}

This is the first study demonstrating profound recent changes in cancer care delivery in multiple centers and the first study modelling overall excess deaths across incident and prevalent cases of cancer (24 sites) where comorbidities were present in $78.1 \%$ of excess deaths.

Near real-time intelligence. There has been a lack of empirical insights and cancer-specific models to counterbalance infectious disease modeling in informing evolving policy responses to the emergency. We propose that weekly national indicators and warnings for vulnerable patient groups such as cancer patients with multimorbidity are essential. For example, weekly cause-specific mortality reporting of registered deaths and linking of those death records to primary and secondary care records for cancer and other services would allow rapid 'root cause analysis' of the extent to which service changes have a net positive or adverse contribution to excess mortality and other non-fatal outcomes.

Changes in cancer services in response to the emergency. We demonstrated major changes in the delivery of cancer services in the UK, which are also likely to impact on cancer patient survival. Two metrics were chosen to reflect both active treatment for patients diagnosed with cancer and the urgent referral of patients who may have a new diagnosis of cancer. The large declines observed in chemotherapy attendance may reflect workforce/capacity or resources being redirected to care for infected patients (e.g., to intensive care) and the desire of clinicians and patients to minimize the risks of COVID-19 infection(8). We note the importance of considering multimorbidity in chemotherapy prioritization, as the risk associated with COVID-19 increases in patients with multimorbidity. However, some of these patients may benefit from chemotherapy avoidance to mitigate immunosuppression. Additionally, we observed large declines in urgent referrals for early cancer diagnosis. Delays in urgent cancer referrals may be caused by patients struggling to secure appointments due to reprioritized health systems, or patients deciding not to seek care due to perceived risk of COVID-19 infection(17). An unintended consequence of these service changes may be excess deaths via increases in emergency admissions, cancer stage shift at presentation and the undermining of curative/life prolonging surgical resection.

Modeling impact of the emergency: the 'untold toll'. We modeled a range of estimates of PAE because the true value is not known. PAE is a summary measure of exposure to the adverse health consequences of the emergency, combining four parameters; the proportion of the population with 1) ill-health in those infected, 2) net adverse health due to changes in health services designed to protect cancer patients from infection, 3) net adverse health consequences of physical distancing, 4) adverse health consequences of economic downturn. Since empirical estimates of each of these four parameters for PAE are not yet available, we chose a PAE range of $10 \%-80 \%$, with $40 \%$ as a plausible estimate.

RIE is a summary measure of the combined impact on mortality of infection, health service change, physical distancing and economic downturn. We modeled a range of estimates of RIE. The only direct evidence we have to date of the magnitude of the RIE comes from national death reporting in England(1). Here, an RIE of 1.5 is consistent with ONS estimates of excess deaths during the COVID-19 emergency in England, which reported almost 8,000 more deaths in the week of 10th April 2020, than the 5-year average of 10,520(1). A higher RIE is possible, given the following evidence: 1) patients with cancer may have 
medRxiv preprint doi: https://doi.org/10.1101/2020.05.27.20083287; this version posted June 1, 2020. The copyright holder for this preprint

(which was not certified by peer review) is the author/funder, who has granted medRxiv a license to display the preprint in perpetuity. It is made available under a CC-BY-NC-ND 4.0 International license .

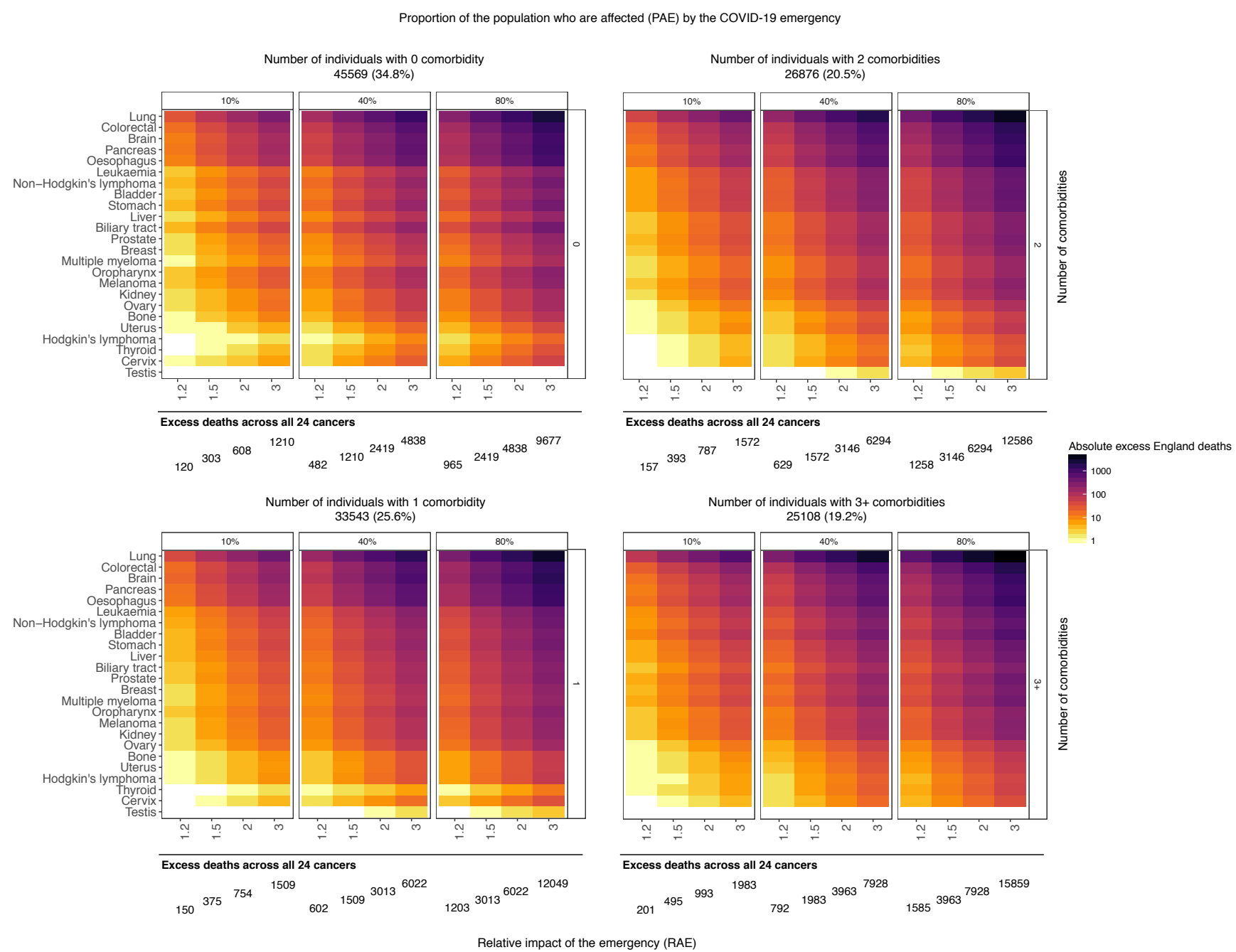

Fig. 5. Estimated number of excess deaths at 1 year due to the COVID-19 emergency by cancer site and number of non-cancer comorbidities for incident cases, scaled up to the population of England aged 30+ consisting of 35 million individuals.

twice the odds of developing COVID-19 infection (odds ratio=2.31) compared to the rest of the population(28), 2) COVID-19 causes patients with cancer to deteriorate more rapidly (hazard ratio $=3.5$ ) compared to those without cancer(11) and 3) COVID-19 case fatality rates are higher in patients with comorbid conditions including CVD, diabetes, hypertension and cancer(29). For cancer patients of working age, unemployment may affect mortality, as we have previously demonstrated across 75 countries(30). Social isolation is also known to represent a mortality risk in cancer $(31,32)$. Our findings relate to predicted excess mortality in the next 12 months. However, the socioeconomic effects on health from the current epidemic are likely to last for a considerable period beyond one year(33), meaning our figures are likely a significant underestimate.

Excess deaths. Based on the available evidence, we estimate an excess of 6,270 excess deaths at 1 year in patients with incident cancers in England. The recorded underlying cause of these excess deaths may be cancer, COVID-19, or comorbidity (such as myocardial infarction), and it is likely that in the COVID-19 emergency, there may be changes in cause-of-death recording. This is why our model uses death from any cause - for which there can be no changes in recording - and which is relevant to multimorbid cancer patients. Our conservative estimate nonetheless represents a significant additional burden of $20 \%$, the total number of cancer deaths annually among incident cases in England is 31,354(34). We present estimates of excess deaths in incident cases, most of whom will be under active surgical, adjuvant or palliative treatments. We also demonstrate excess deaths in those with prevalent cancers, many of whom have survived the initial high-risk period; these data are particularly relevant to ongoing patient management.

Model application in the US. Using SEER estimates of incidence and 1-year mortality, we modeled excess deaths in the US. For example, with an RIE 1.5 and PAE $40 \%$ we estimate 33,890 excess 1-year deaths. Like other cancer registries, SEER does not obtain information on the range of (COVID-19 relevant) comorbidities. We found good agreement between Eng- 
land and US estimates for incidence and mortality(35).

Importance of multimorbidity. We show that most patients with cancer have non-cancer comorbid conditions which confer additional mortality risk; many of these comorbidities are treatable by non-cancer services. The COVID-19 emergency has prompted new questions about which cancer patients are most vulnerable and how best to mitigate risk. Effective management of hypertension, for example, may avert death and major adverse events, but it is possible that blood pressure control may worsen during the emergency and contribute to excess deaths.

Policy implications. Our study can inform policy in four areas. First, there is a need to mobilize access to real-time national data on mortality (to determine which disease combinations pose the greatest risk) and on cancer health services activity (to monitor the effects of system change during the emergency on care delivery and future health outcomes). This health services intelligence should include both data from cancer services and from those services (e.g. internists and family physicians) who manage the treatable comorbidities of cancer patients. Second, the triaging of chemotherapy prioritization would usefully incorporate patient-specific risk/benefit assessments to include multimorbidity, particularly in situations where chemotherapy outweighs the benefits of non-treatment and safety issues(17) related to COVID-19. Third, there is a need to enable access to life-saving early diagnosis and to apply a triage system to prioritize urgent referrals for citizens with worrying symptoms, especially those with comorbidities, as multimorbidity may mask an underlying cancer. Fourth, the policy of 'shielding' vulnerable patients under active treatment for cancer, or with one of a range of other non-malignant conditions has been implemented in 1.5 million individuals in England, involving home delivery of food parcels and medicines for an initial period of 12 weeks. We propose that estimates of background (pre COVID-19) 1-year mortality risks provides a transparent rationale, readily executable in health records, for identifying priority patient groups including cancer patients with multimorbidities to receive shielding interventions.

Limitations. This study has important limitations. First, there is a lack of near real-time clinical data in multiple digitally-mature hospitals with large numbers of COVID-19 infected patients. Second, the health records we used may have missed cases of cancer and underestimated incidence(36); if so, our estimates of excess deaths may be conservative. The National Health Service has national linked hospital admissions and cancer registration data with information on stage and details of surgical, chemotherapeutic and radiotherapy treatment of cancer. However, information governance for such data can take months to secure, making data-enabled research and timesensitive responsive service improvement difficult. Third, we did not have access to multimorbidity data for the
US. Fourth, we did not have access to data on children.

\section{Conclusion}

Our data have highlighted how cancer patients with multimorbidity are a particularly at-risk group during the current pandemic. In order to ensure effective cancer policy and avoid excess deaths, both during and after the COVID-19 emergency, it is critical to ensure near-real time reporting of cause-specific excess mortality, urgent cancer referrals and treatment statistics, so as to inform the most optimal delivery of care in this extremely vulnerable group of patients.

\section{Authors' contribution}

Research question: $\mathrm{AL}, \mathrm{HH}$. Funding: $\mathrm{AL}, \mathrm{AB}, \mathrm{HH}$, DATA-CAN. Study design and analysis plan: AL, LP, AB, MK, WHC, HH. Preparation of data, including electronic health record phenotyping in the CALIBER open portal: AL, LP, SD. Provision of weekly hospital data: GH, KPJ, MDF, DH, ML, KB, CD. Statistical analysis: AL, LP, MK, WHC. Drafting initial versions of manuscript: $\mathrm{AL}, \mathrm{HH}$. Drafting final versions of manuscript: $\mathrm{AL}, \mathrm{ML}, \mathrm{HH}$. Critical review of early and final versions of manuscript: All authors.

\section{Declaration of interests}

ML has received honoraria from Pfizer, EMD Serono and Roche for presentations unrelated to this research. ML has received an unrestricted educational grant from Pfizer for research unrelated to the research presented in this paper. MDF has received research funding from AstraZeneca, Boehringer Ingelheim, Merck and MSD and honoraria from Achilles, AstraZeneca, Bayer, Boehringer Ingelheim, Bristol-Meyers Squibb, Celgene, Guardant Health, Merck, MSD, Nanobiotix, Novartis, Pharmamar, Roche and Takeda for advisory roles or presentations unrelated to this research. GRF receives funding from companies that manufacture drugs for hepatitis C virus (AbbVie, Gilead, MSD) and consult for GSK and Arbutus in areas unrelated to this research.

\section{Acknowledgements}

We thank Tony Hagger, Shiva Thapa, Mohammed Emran, Cara Anderson, Louise Herron, Philip Melling and Lee Cogger for their help on collating data on urgent cancer referrals and chemotherapy attendances. We thank Charles Swanton for his valuable comments on the manuscript. This work uses data provided by patients and collected by the NHS as part of their care and support.

\section{Funding statement}

We acknowledge Health Data Research UK (HDR UK) support for the HDR UK substantive sites involved in this research (HDR London, HDR Wales and Northern Ireland) and DATA-CAN. DATA-CAN is part of the Digital Innovation 
Hub Programme, delivered by HDR UK and funded by UK Research and Innovation through the government's Industrial Strategy Challenge Fund (ISCF). AGL is supported by funding from the Wellcome Trust, National Institute for Health Research (NIHR) University College London Hospitals and NIHR Great Ormond Street Hospital Biomedical Research Centers. AB is supported by research funding from NIHR, British Medical Association, Astra-Zeneca and UK Research and Innovation. KPJ is supported by the NIHR Great Ormond Street Hospital Biomedical Research Centre. CD is funded by UCLPartners. HH is an NIHR Senior Investigator and is funded by the NIHR University College London Hospitals Biomedical Research Centre, supported by Health Data Research UK (grant No. LOND1), which is funded by the UK Medical Research Council, Engineering and Physical Sciences Research Council, Economic and Social Research Council, Department of Health and Social Care (England), Chief Scientist Office of the Scottish Government Health and Social Care Directorates, Health and Social Care Research and Development Division (Welsh Government), Public Health Agency (Northern Ireland), British Heart Foundation, Wellcome Trust, The BigData@Heart Consortium, funded by the Innovative Medicines Initiative-2 Joint Undertaking under grant agreement No. 116074.

\section{Supplementary methods}

This study was performed as part of the CALIBER program (https://caliberresearch.org/portal), which is a research resource consisting of anonymized, coded variables extracted from linked electronic health records, methods, tools and specialized infrastructure. Ethical approval was granted (20_074R2) by the MHRA (UK) Independent Scientific Advisory Committee of the Clinical Practice Research Data Link under Section 251 (NHS Social Care Act 2006). The interpretation and conclusions contained in this study are those of the authors' alone.

15 comorbid conditions or condition clusters involving 40 individual conditions defined by the Centers for Disease Control (CDC) or Public Health England (PHE) as associated risk factors for poor outcomes caused by severe COVID-19 infection. Both lists from CDC and PHE included chronic respiratory disease; chronic heart disease; immunocompromised; HIV or use of corticosteroids; obesity; diabetes; chronic kidney disease; chronic liver disease. The PHE list included additional condition clusters (chronic neurological disorders; splenic disorders). We have performed analyses for all the above conditions and have additionally considered hypertension, Crohn's disease, cystic fibrosis and rheumatoid arthritis.

Given that condition clusters such as (i) chronic heart disease would involve a range of conditions, we have derived composite variables to include 15 conditions considered as cardiovascular disease (CVD) that included acute myocardial infarction, unstable angina, chronic stable angina, heart failure, cardiac arrest or sudden coronary death, transient ischemic attack, intracerebral hemorrhage, subarachnoid hemorrhage, ischemic stroke, abdominal aortic aneurysm, peripheral arterial disease, atrial fibrillation, congenital heart disease, hypertrophic and dilated cardiomyopathy and valve disease (multiple, mitral and aortic)(23). We also considered (ii) Hypertension, defined as $\geq 140 \mathrm{mmHg}$ systolic blood pressure (or $\geq 150 \mathrm{mmHg}$ for people aged $\geq 60$ years without diabetes and chronic kidney disease) and/or $\geq 90 \mathrm{mmHg}$ diastolic blood pressure(22), (iii) type 2 diabetes, (iv) obesity, defined as a body mass index of $\geq 30 \mathrm{~kg} / \mathrm{m} 2$, (v) chronic kidney disease (CKD), (vi) chronic obstructive pulmonary disease (COPD)(25), (vii) patients on immunosuppressive drugs (not cancer chemotherapy), (viii) patients with HIV or corticosteroid prescription, (ix) chronic neurological disorders, defined as a composite of Parkinson's disease, motor neuron disease, learning disability and cerebral palsy, (x) multiple sclerosis separately, (xi) splenic disorders, defined as a composite of splenomegaly, splenectomy and hyposplenism, (xii) chronic liver diseases, defined as a composite of chronic viral hepatitis B or C, primary biliary cholangitis, liver fibrosis, liver cirrhosis and non-alcoholic fatty liver disease, (xiii) Crohn's disease, (xiv) cystic fibrosis and (xv) rheumatoid arthritis(24).

Analyses. In CALIBER (England), we estimated the frequency (\%) of comorbid conditions in prevalent cancers (diagnosed at or any time prior to baseline) and incident cancers as occurring any time over follow-up. We estimated incidence rates for as the number of new cancers by cancer site per 100,000 person years. We compared CALIBER incidence rates for England with those from the UK from the International Agency for Research on Cancer (IARC). In CALIBER, we generated Kaplan-Meier estimates on 1year mortality for incident cancers observed from 2012-2016 (by cancer cites and by number of comorbid conditions) and prevalent cancers (by cancer sites and by number of comorbid conditions). We only considered non-fatal incident cases, i.e., alive for at least 30 days following cancer diagnosis to account for potential time lag in death recording. Excess deaths for incident cancers were estimated based on 1-year mortality and 1-year incidence rates, scaled up to a population of 35,407,313 individuals aged 30 and above in England based on population estimates from 2018(37). We used publicly available data from the US from the Surveillance, Epidemiology, and End Results (SEER) program to estimate 1 year mortality as 1 minus the reported survival(27). We considered mortality data from SEER for individuals aged 40-64, 65-74 and 75+. We applied our model (RIE and PAE) to SEER estimates of incidence and 1-year mortality (according to the corresponding age groups) scaled up to the US population of individuals aged 40-64 (208,284,801 individuals) and 65-74 (61,346,445 individuals) based on population estimates from 2018(38).

\section{Bibliography}

1. Office for National Statistics. Deaths registered weekly in England and Wales, provisional: week ending 10 April 2020. Accessed 22 April 2020. 
medRxiv preprint doi: https://doi.org/10.1101/2020.05.27.20083287; this version posted June 1, 2020. The copyright holder for this preprint (which was not certified by peer review) is the author/funder, who has granted medRxiv a license to display the preprint in perpetuity. It is made available under a CC-BY-NC-ND 4.0 International license .

2. Lisa Rosenbaum. The Untold Toll — The Pandemic's Effects on Patients without Covid-19. New England Journal of Medicine. doi: 10.1056/NEJMms2009984.

3. Eduard Vrdoljak, Richard Sullivan, and Mark Lawler. Cancer and COVID-19; how do we manage cancer optimally through a public health crisis? European Journal of Cancer, 132: P98-99, 2020.

4. American Cancer Society. Survey: COVID-19 Affecting Patients' Access to Cancer Care. Accessed 19 April 2020

5. American College of Surgeons. COVID-19 Guidelines for Triage of Breast Cancer Patients. Accessed 19 April 2020.

6. American Society for Radiation Oncology. COVID-19 Coding Guidance. Accessed 19 April 2020.

7. British Society of Gastroenterology. Endoscopy activity and COVID-19: BSG and JAG guidance. Accessed 19 April 2020.

8. National Institute for Health and Care Excellence. COVID-19 rapid guideline: delivery of systemic anticancer treatments. Accessed 19 April 2020.

9. Talha Khan Burki. Cancer guidelines during the COVID-19 pandemic. The Lancet Oncology, 2020.

10. David J Thomson, David Palma, Matthias Guckenberger, Panagiotis Balermpas, Jonathan J Beitler, Pierre Blanchard, David Brizel, Wilfred Budach, Jimmy Caudell, June Corry, and Others. Practice recommendations for risk-adapted head and neck cancer radiotherapy during the COVID-19 pandemic: an ASTRO-ESTRO consensus statement. International Journal of Radiation Oncology* Biology* Physics, 2020.

11. Wenhua Liang, Weijie Guan, Ruchong Chen, Wei Wang, Jianfu Li, Ke Xu, Caichen Li, Qing Ai, Weixiang Lu, Hengrui Liang, and Others. Cancer patients in SARS-CoV-2 infection: a nationwide analysis in China. The Lancet Oncology, 21(3):335-337, 2020.

12. Hanping Wang and Li Zhang. Risk of COVID-19 for patients with cancer. The Lancet Oncology, 21(4):e181, 2020

13. Centers for Disease Control and Prevention. Groups at Higher Risk for Severe IIIness. Accessed 19 April 2020

14. Public Health England. Guidance on shielding and protecting people defined on medical grounds as extremely vulnerable from COVID-19. Accessed 19 April 2020.

15. Public Health England. Guidance on social distancing for everyone in the UK. Accessed 19 April 2020.

16. Cristina Renzi, Aradhna Kaushal, Jon Emery, Willie Hamilton, Richard D. Neal, Bernard Rachet, Greg Rubin, Hardeep Singh, Fiona M. Walter, Niek J. de Wit, and Georgios Lyratzopoulos. Comorbid chronic diseases and cancer diagnosis: disease-specific effects and underlying mechanisms. Nature Reviews Clinical Oncology, 2019. ISSN 1759-4774. doi: 10.1038/s41571-019-0249-6.

17. Timothy P Hanna, Gerald A Evans, and Christopher M Booth. Cancer, COVID-19 and the precautionary principle: prioritizing treatment during a global pandemic. Nature Reviews Clinical Oncology, pages 1-3, 2020

18. Amitava Banerjee, Laura Pasea, Steve Harris, Arturo Gonzalez-Izquierdo, Ana Torralbo, Laura Shallcross, Mahdad Noursadeghi, Deenan Pillay, Christina Pagel, Wai Keong Wong, and Others. Estimating excess 1 -year mortality from COVID-19 according to underlying conditions and age in England: a rapid analysis using NHS health records in 3.8 million adults. Lancet (in press), 2020

19. Health Data Research UK. DATA-CAN - The Health Data Research Hub for Cancer. Accessed 20 April 2020

20. Spiros C Denaxas, Julie George, Emily Herrett, Anoop D Shah, Dipak Kalra, Aroon D Hingorani, Mika Kivimaki, Adam D Timmis, Liam Smeeth, and Harry Hemingway. Data resource profile: cardiovascular disease research using linked bespoke studies and electronic health records (CALIBER). International journal of epidemiology, 41(6):1625-1638, 2012.

21. Spiros Denaxas, Arturo Gonzalez-Izquierdo, Kenan Direk, Natalie K Fitzpatrick, Ghazaleh Fatemifar, Amitava Banerjee, Richard J B Dobson, Laurence J Howe, Valerie Kuan, $\mathrm{R}$ Tom Lumbers, and Others. UK phenomics platform for developing and validating electronic health record phenotypes: CALIBER. Journal of the American Medical Informatics Association, 2019

22. Eleni Rapsomaniki, Adam Timmis, Julie George, Mar Pujades-Rodriguez, Anoop D. Shah, Spiros Denaxas, Ian R. White, Mark J. Caulfield, John E. Deanfield, Liam Smeeth, Bryan Williams, Aroon Hingorani, and Harry Hemingway. Blood pressure and incidence of twelve cardiovascular diseases: Lifetime risks, healthy life-years lost, and age-specific associations in 1.25 million people. The Lancet, 383(9932):1899-1911, 2014. ISSN 1474547X. doi: 10.1016/S0140-6736(14)60685-1.

23. Anoop Dinesh Shah, Claudia Langenberg, Eleni Rapsomaniki, Spiros Denaxas, Mar Pujades-Rodriguez, Chris P. Gale, John Deanfield, Liam Smeeth, Adam Timmis, and Harry Hemingway. Type 2 diabetes and incidence of cardiovascular diseases: A cohort study in 1.9 million people. The Lancet Diabetes and Endocrinology, 3(2):105-113, 2015. ISSN 22138595. doi: 10.1016/S2213-8587(14)70219-0.

24. Mar Pujades-Rodriguez, Bram Duyx, Sara L Thomas, Dimitris Stogiannis, Anisur Rahman, Liam Smeeth, and Harry Hemingway. Rheumatoid arthritis and incidence of twelve initia presentations of cardiovascular disease: a population record-linkage cohort study in England. PloS one, 11(3), 2016.

25. Maria Pikoula, Jennifer Kathleen Quint, Francis Nissen, Harry Hemingway, Liam Smeeth, and Spiros Denaxas. Identifying clinically important COPD sub-types using data-driven approaches in primary care population based electronic health records. BMC medical informatics and decision making, 19(1):86, 2019.

26. Valerie Kuan, Spiros Denaxas, Arturo Gonzalez-Izquierdo, Kenan Direk, Osman Bhatti, Shanaz Husain, Shailen Sutaria, Melanie Hingorani, Dorothea Nitsch, Constantinos A Parisinos, R Thomas Lumbers, Rohini Mathur, Reecha Sofat, Juan P Casas, lan C K Wong, Harry Hemingway, and Aroon D Hingorani. A chronological map of 308 physical and mental health conditions from 4 million individuals in the English National Health Service. The Lancet Digital Health, 1(2):e63-e77, 2019. ISSN 25897500. doi: 10.1016/S2589-7500(19) 30012-3.

27. MA Hernan and JM Robins. Causal Inference: What If. Boca Raton: Chapman \& Hall/CRC.,

28. Jing Yu, Wen Ouyang, Melvin L K Chua, and Conghua Xie. SARS-CoV-2 transmission in patients with cancer at a tertiary care hospital in Wuhan, China. JAMA oncology, 2020

29. Zunyou Wu and Jennifer M McGoogan. Characteristics of and important lessons from the coronavirus disease 2019 (COVID-19) outbreak in China: summary of a report of 72314 cases from the Chinese Center for Disease Control and Prevention. Jama, 2020.

30. Mahiben Maruthappu, Johnathan Watkins, Aisyah Mohd Noor, Callum Williams, Raghib Ali, Richard Sullivan, Thomas Zeltner, and Rifat Atun. Economic downturns, universal health coverage, and cancer mortality in high-income and middle-income countries, 1990-2010: a longitudinal analysis. The Lancet, 388(10045):684-695, 2016.

31. Candyce H Kroenke, Laura D Kubzansky, Eva S Schernhammer, Michelle D Holmes, and Ichiro Kawachi. Social networks, social support, and survival after breast cancer diagnosis. J Clin Oncol, 24(7):1105-1111, 2006

32. Marko Elovainio, Christian Hakulinen, Laura Pulkki-Råback, Marianna Virtanen, Kim Josefsson, Markus Jokela, Jussi Vahtera, and Mika Kivimäki. Contribution of risk factors to excess mortality in isolated and lonely individuals: an analysis of data from the UK Biobank cohort study. The Lancet Public Health, 2(6):e260-e266, 2017.

33. Institute for Fiscal Studies. Accessed 24 April 2020.

34. Cancer Research UK. Cancer Statistics for the UK. Accessed 20 April 2020.

35. Surveillance, Epidemiology, and End Results (SEER) Program. Overview of the SEER Program. Accessed 19 April 2020.

36. Andrea V Margulis, Joan Fortuny, James A Kaye, Brian Calingaert, Maria Reynolds, Este Plana, Lisa J McQuay, Willem Jan Atsma, Billy Franks, Stefan de Vogel, and Others. Validation of cancer cases using primary care, cancer registry, and hospitalization data in the United Kingdom. Epidemiology (Cambridge, Mass.), 29(2):308, 2018.

37. Office for National Statistics. Estimates of the population for the UK, England and Wales, Scotland and Northern Ireland. Accessed 23 April 2020.

38. United States Census Bureau. National Population by Characteristics: 2010-2019. Accessed 23 April 2020. 